\title{
Lapurdum
}

Euskal ikerketen aldizkaria | Revue d'études basques |

Revista de estudios vascos | Basque studies review

$7 \mid 2002$

Numéro VII

Recensement1999

\section{La langue basque au Pays Basque : diagnostic, évolution et prospective}

\section{Eguzki Urteaga}

\section{(2) OpenEdition \\ 1 Journals}

Édition électronique

URL : http://journals.openedition.org/lapurdum/1036

DOI : 10.4000/lapurdum.1036

ISSN : 1965-0655

Éditeur

IKER

Édition imprimée

Date de publication : 1 octobre 2002

Pagination : 227-338

ISBN : 2-86781-321-2

ISSN : $1273-3830$

Référence électronique

Eguzki Urteaga, «La langue basque au Pays Basque : diagnostic, évolution et prospective », Lapurdum [En ligne], 7 | 2002, mis en ligne le 01 juin 2009, consulté le 30 avril 2019. URL : http:// journals.openedition.org/lapurdum/1036; DOI : 10.4000/lapurdum.1036 


\section{La langue basque au Pays Basque : diagnostic, évolution et prospective}

Eguzki URTEAGA

Docteur en sociologie Université de Bordeaux 2

\section{Introduction}

L'enquête Etude de l'histoire familiale de 1999 a été conçue avec le concours de l'Institut National d'Etudes Démographiques et réalisée par I'INSEE. Elle fait l'objet d'une exploitation concertée entre les deux institutions. Pour la première fois, elle comportait un volet consacré à la "Transmission familiale des langues et parlers". Les questions concernant cette transmission ont permis d'isoler et d'étudier les trois grands axes suivants :

- d'une part, "l'héritage" de ces différentes langues transmises par nos parents, considéré isolément, dans notre enfance,

- d'autre part, l'usage que nous-mêmes avons adopté vis-à-vis de ces langues, avec nos jeunes enfants,

- et enfin, notre pratique actuelle avec notre entourage, de ces mêmes langue.

\section{Locuteurs bascophones}

\begin{tabular}{|c|c|c|c|c|c|c|c|}
\hline \multicolumn{7}{|c|}{ Nombre de locuteurs adultes parlant le basque } \\
\hline \multirow{3}{*}{ Années } & Habituellement & \multicolumn{2}{c|}{ Occasionnellement } & \multicolumn{2}{c|}{ Jamais } & \multirow{2}{*}{ Population } \\
\cline { 2 - 7 } & Effectifs & $\%$ & Effectifs & $\%$ & Effectifs & $\%$ & $1187 !$ \\
1915 & 4664 & 39,29 & 505 & 4,25 & 6702 & 56,46 & 13985 \\
1920 & 5793 & 41,42 & 866 & 6,19 & 7326 & 52,38 & 15204 \\
1925 & 5582 & 36,72 & 768 & 5,05 & 8854 & 58,23 & 17671 \\
1930 & 6678 & 37,79 & 1024 & 5,79 & 9969 & 56,42 & 15791 \\
1945 & 5259 & 33,30 & 1085 & 6,87 & 9447 & 59,83 & 12144 \\
1945 & 3392 & 27,93 & 840 & 6,92 & 7912 & 65,15 & 19568 \\
1950 & 5125 & 26,19 & 1019 & 5,21 & 13424 & 68,60 & 20210 \\
1955 & 6483 & 33,08 & 1232 & 6,10 & 12495 & 61,83 & 17610 \\
1960 & 3592 & 20,40 & 1312 & 7,45 & 12706 & 72,15 & 20716 \\
1965 & 3968 & 23,98 & 1186 & 5,72 & 14562 & 70,29 & 17690 \\
1970 & 3626 & 20,50 & 1279 & 7,23 & 12785 & 72,27 & 17181 \\
1975 & 2797 & 22,10 & 978 & 5,69 & 12406 & 72,21 & 12896 \\
1980 & 650 & 21,56 & 1381 & 10,71 & 8734 & 67,73 & 2266 \\
Total & 62330 & 26,02 & 38 & 1,69 & 1638 & 72,29 & 214803 \\
\hline
\end{tabular}

Source : Insee - Enquête 1999 Etude de l'histoire familiale 
Ces questionnaires, remplis en même temps que les bulletins du recensement de mars 1999, ont concerné 380000 adultes vivant en Métropole (dont environ 6000 au Pays Basque), dont les plus âgés sont nés avant la guerre de 1914. En ce qui concerne les langues régionales ou minoritaires et afin d'obtenir une représentativité plus proche de la réalité, certaines aires géographiques, notamment au Pays Basque, ont été surévaluées dans l'échantillon et les résultats redressés avant publication. Les pondérations détaillées au niveau régional, ont également permis une analyse plus fine par catégorie socioprofessionnelle, âge, sexe et tranche d'unité urbaine.

À partir de ces donnécs, nous avons réalisé un commentaire se subdivisant en trois phases:

1) l'état des lieux, c'est-à-dire la lecture des données recueillies;

2) l'évolution, à savoir les modifications ou permanences constatées au fil du temps;

3) la prospective ou les conclusions pouvant être tirées pour l'avenir.

Nous avançons l'hypothèse selon laquelle, après une période de déclin plus ou moins continu, la langue basque semble en voie de stabilisation, voire de réappropriation sous certains aspects tels que la transmission familiale occasionnelle. Or, pour qu'il en soit ainsi à l'avenir des mesures structurelles de développement de la langue basque doivent être adoptées.

\section{Pratique linguistique globale}

1) Au Pays Basque, à savoir, dans l'arrondissement de Bayonne et les deux cantons souletins de Mauléon et Tardets-Sorholus, l'on dénombre 63000 locuteurs adultes bascophones sur une population de 213000 habitants de 18 ans et plus. Cela représente $29,57 \%$ de la population adulte du territoire concerné. Auxquels s'ajoutent un peu plus de 13500 locuteurs occasionnels $(6,34 \%)$. Ceci dit, parmi ces 76513 locuteurs habituels ou occasionnels, il est malaisé de distinguer les bilingues actifs et passifs, c'est-à-dire ceux qui pratiquent bien, voire très bien le basque et ceux qui parlent cette langue avec difficulté.

2) La pratique de la langue basque par génération montre une stabilisation de la pratique habituelle du basque et une progression de la pratique occasionnelle auprès de générations nées entre 1965 et 1980, autrement dit auprès des personnes ayant entre 34 et 19 ans au moment du recensement. En effet, la pratique habituelle de la langue basque par les enfants diminue de moitié entre les générations nées en 1920 et en 1955 , passant de $41 \%$ à $20 \%$, puis se stabilise, voire progresse légèrement. La réception occasionnelle, pour sa part, augmente de manière significative dans la mesure où, après plusieurs décennies de stagnation, elle croît fortement en cinq ans au sein des jeunes générations, allant de $5,69 \%$ à près de $11 \%$. 
Si la progression de la pratique observée chez les personnes nées en 1920 et 1950 est imputable à la fin des guerres mondiales et à l'essor de la natalité, il en est autrement pour les jeunes générations. En effet, cette constance, voire ce progrès, sont en lien avec le souci croissant des parents, particulièrement ceux dont les enfants sont scolarisés dans l'enseignement bilingue, de parler le basque à leurs enfants. À la fois par souci de transmettre cette langue à laquelle ils sont fortement attachés et par volonté d'aider leurs enfants dans leur scolarité. Ce changement d'attitude prend d'autant plus d'ampleur avec le développement de l'enseignement du et en langue basque. Ainsi, dans le seul primaire, entre 1993 et 2001 , les effectifs sont passés de 1309 à 2600 dans le public bilingue, de 593 à 1146 dans le privé bilingue et de 1005 à 1393 dans l'associatif bilingue. Auxquels s'ajoutent les enfants apprenant la langue basque 1 à 3 heures par jour en initiation ou en option.

\section{Locuteurs basques par catégories socioprofessionnelles}

\begin{tabular}{|c|c|c|c|c|c|c|c|c|}
\hline \multirow{2}{*}{$\begin{array}{l}\text { Catégories } \\
\text { socioprofessionnelles } \\
\begin{array}{l}\text { (retraités classés dans } \\
\text { leurs ancienne CS) }\end{array}\end{array}$} & \multicolumn{2}{|c|}{ Population } & \multicolumn{3}{|c|}{ Non bascophones } & \multicolumn{3}{|c|}{ Bascophones } \\
\hline & Effectifs & $\%$ & Effectifs & Taux & $\%$ & Effectifs & Taux & $\%$ \\
\hline Agriculteurs & 23593 & 10,97 & 3116 & 13,21 & 2,04 & 20477 & 86,79 & 32,62 \\
\hline $\begin{array}{l}\text { Artisans, commerçants, } \\
\text { chefs d'E. }\end{array}$ & 14311 & 6,65 & 10277 & 71,82 & 6,75 & 4033 & 28,18 & 6,43 \\
\hline Prof. libérales et cadres & 13873 & 6,45 & 12557 & 90,51 & 8,24 & 1316 & 9,49 & 2,10 \\
\hline Prof. intermédiaires & 29399 & 13,67 & 23680 & 80,55 & 15,54 & 5719 & 19,45 & 9,11 \\
\hline Employés & 56960 & 26,48 & 46199 & 81,11 & 30,32 & 10761 & 18,89 & 17,14 \\
\hline Ouvriers & 37630 & 17,49 & 26516 & 70,47 & 17,40 & I1 114 & 29,53 & 17,70 \\
\hline Jamais travaillé & 31470 & 14,63 & 23929 & 76,04 & 15,71 & 7540 & 23,96 & 12,01 \\
\hline Inconnu & 7905 & 3,67 & 60093 & 77,07 & 4,00 & 1813 & 22,95 & 2,89 \\
\hline Ensemble & 215140 & 100 & 152367 & 70,82 & 100 & 62773 & 29,18 & 100 \\
\hline
\end{tabular}

Source : Insee - Enquête 1999 Etude de l'histoire familiale

3) Dans un avenir plus ou moins proche, l'on s'achemine vers une constance puis une légère réappropriation de la langue basque. La remontée amorcée dans la pratique occasionnelle parmi les jeunes générations abonde dans ce sens. Toutefois, cette remontée est fragile et reste conditionnée à la mise en oeuvre d'une politique linguistique de promotion et de soutien à la langue basque. Effectivement, le déclin des anciens acteurs de la transmission dite naturelle tels que la famille, la communauté villageoise ou l'Église exige le développement de nouveaux vecteurs de transmission, en particulier l'école, les médias et les services publics.

\section{Pratique linguistique par sexe}

1) Parmi les près de 63000 locuteurs bascophones du Pays Basque, une faible majorité sont des hommes $(52,10 \%)$ puisque l'on dénombre 32706 hommes pour 30067 femmes. Cette plus forte tendance des hommes à pratiquer le basque s'explique, en partie, par la sureprésentation du "sexe fort" dans le monde du travail et dans les métiers traditionnels tels que l'agriculture, la pêche et l'artisanat où la langue basque est largement pratiquée. 
2) L'écart entre hommes et femmes pour ce qui est de la pratique linguistique s'est amenuisé au cours des demières décennies. Et ce, dans la mesure où les femmes ont peu à peu rejeté le sentiment de honte et d'inutilité sociale associé à la langue basque, sous l'effet de la féminisation du système scolaire et du monde du travail. En effet, plus que les hommes, les femmes ont longtemps intériorisé l'idée selon laquelle le basque était une langue du passé, ancrée dans la ruralité et la tradition. Dès lors, la seule manière de sortir de leur village, de leur milieu social et d'embrasser la modemité était d'apprendre le français dont la maîtrise permettait de devenir employée, institutrice, voire médecin.

3) L'on peut avancer, sans trop oser se tromper, que cette aptitude sera de plus en plus présente chez les femmes. Car, au fur et à mesure que le marché du travail se féminisera et que le nombre d'emplois exigeant la maîtrise du basque se développera dans les secteurs dans lesquels la gente féminine est prépondérante, à l'image de l'enseignement et de la culture, l'on aboutira a une parité parmi les locuteurs bascophones.

\section{Pratique linguistique par catégorie socioprofessionnelle}

1) La pratique linguistique diffère suivant la catégorie socioprofessionnelle (CS) avec une sureprésentation parmi les agriculteurs $(32,62 \%)$, les employés $(17,14 \%)$ et les ouvriers $(17,70 \%)$. Ces trois catégories constituent plus des deux tiers des bascophones $(67,47 \%)$. Viennent ensuite les professions intermédiaires, les artisans, commerçants et chefs d'entreprises et, dans une moindre mesure, les cadres et professions libérales dont uniquement $2,10 \%$ pratiquent le basque.

La répartition est analogue lorsque l'on se réfère au taux de bascophonie par CS puisque près de $87 \%$ des agriculteurs parlent le basque suivis, à une certaine distance, par les ouvriers et les artisans, commerçants et chefs d'entreprise qui sont pratiquement trois sur dix à utiliser cette langue. Puis, l'on trouve les personnes n'ayant jamais travaillé et dont la majorité sont soit élèves soit étudiants. Les professions intermédiaires et les employés sont relativement proches, contrairement aux professions libérales et cadres qui ne sont que $9,5 \%$ à pratiquer le basque.

Cette désaffection des professions libérales et intellectuelles pour la langue basque est étroitement associée à la faible utilité sociale accordée au basque. Ainsi, les classes moyennes et supérieures rechignent à "investir" dans le basque car il n'en attendent aucun gain dans les domaines scolaires et professionnels. Ils préfèrent parler l'anglais $(18,06 \%)$ et, dans une moindre mesure, l'espagnol $(9,88$ $\%)$.

2) Cela met en exergue une évolution progressive de la répartition des bascophones par CS. Effectivement, la part des agriculteurs et des ouvriers décline en valeur absolue puisque ces catégories ont vu leurs effectifs fondre au cours des dernières décennies pour ne plus représenter que $11 \%$ et $17,5 \%$ de la population du Pays Basque. En revanche, les employés et les professions intermédiaires croissent car plus d'un salarié sur quatre est employé et près de $14 \%$ des actifs sont cadres moyens. 
La crise de l'agriculture ou la fin du monde paysan n'est pas étrangère à cet état de fait. En effet, le nombre d'actifs vivant de la production et de la vente de produits agricoles a fortement chuté depuis le lendemain de la seconde guerre mondiale et, surtout, depuis le milieu des années 60 . L'on a observé à la fois une diminution et une concentration du nombre d'exploitations. Les petits producteurs ont été les premières victimes de ces mutations, même si, sous l'effet de la mobilisation des syndicats agricoles, le déclin a été moins sévère que dans d'autres régions.

3) $\mathrm{Au}$ regard des changements économiques, démographiques et sociolinguistiques, l'on peut considérer que les bascophones appartiendront de plus en plus aux professions intermédiaires et seront en nombre croissant, bien que ce soit dans une moindre mesure, parmi les cadres supérieurs, particulièrement dans les métiers de l'enseignement, de l'information et de la communication. D'autant que plusieurs milliers de jeunes se déplacent, chaque année, dans les principales agglomérations de la Région Aquitaine pour y suivre des études universitaires souvent prolongées.

\section{Pratique linguistique par tranche d'unité urbaine}

Transmission de la langue basque par génération

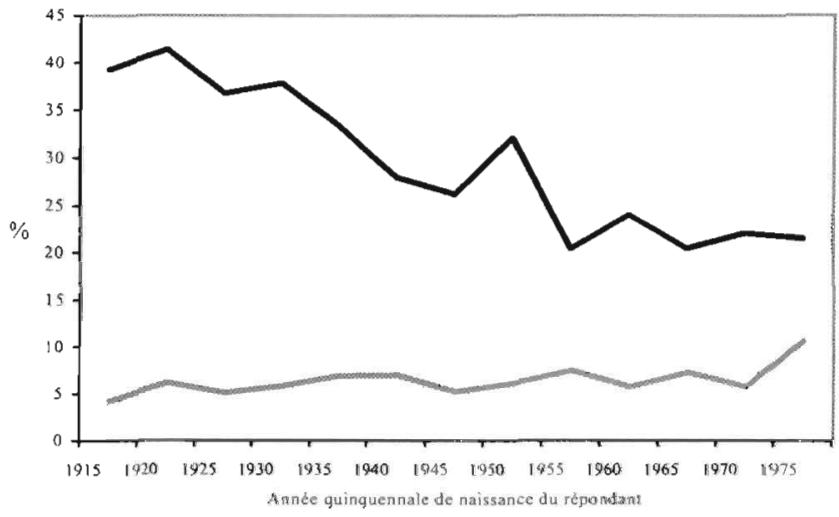

SOURCE : Insee

Enquête 1999

Etude de l'histoire familiale

1) La langue basque est majoritairement pratiquée dans des communes rurales puisque, en Aquitaine, sept personnes sur dix résident dans des communes de petite taille qui se retrouvent majoritairement dans le Pays Basque intérieur. En effet, si en Basse-Navarre et en Soule les bascophones représentent plus de la moitié de la population, le chiffre décline dans le Labourd intérieur et plus encore dans l'agglomération bayonnaise. 
Or, de nombreux locuteurs bascophones habitent dans des zones urbaines et dans des communes dont la population oscille entre 20000 et 200000 personnes dans la mesure où 3474 personnes $(4,70 \%$ ) vivent dans des villes ayant entre 20 000 et 100000 habitants, 16448 individus $(22,29 \%)$ résident dans des agglomérations ayant entre 100000 et 200000 habitants et 2113 personnes $(2,86$ $\%$ ) ont leur logement principal dans des agglomérations de plus de 200000 habitants. Cela signifie que près de $30 \%$ de la population de la région vit dans des communes de plus de 20000 habitants.

2) Cela indique une urbanisation progressive des bascophones qui est à mettre en relation avec la désertification rurale. Effectivement, un nombre croissant d'habitants du Pays Basque intérieur se déplace vers la zone côtière où se trouvent les principales villes. Ainsi, la communc d'Anglet a vu sa population croître fortement au cours de la dernière décennie. Simultanément, cette urbanisation des locuteurs bilingues est corrélée au flux migratoire constant en direction des principales agglomérations aquitaines, paloises et bordelaises surtout. En effet, nombreux sont les étudiants et les actifs qualifiés à quitter le Pays Basque en l'absence d'une Université de plein exercice et consécutivement au faible nombre d'emplois qualifiés et très qualifiés sur le plan local.

3) À l'avenir, l'on peut imaginer que cette proportion croitra avec l'élévation du niveau d'étude. De la sorte, bien que plus faibles démographiquement, les nouvelles classes d'âges seront proportionnellement plus nombreuses à poursuivre des études dans l'enseignement supérieur dont l'offre fait défaut au Pays Basque. Ainsi, la faculté de Bayonne n'est qu'une annexe de l'Université de Pau et des Pays de l'Adour et ne propose que certains enseignements ayant trait, notamment aux Sciences Economiques, au Droit, aux Etudes Basques ou aux Lettres Modernes.

\section{Transmission de la langue basque}

1) La transmission familiale du basque résiste assez bien, particulièrement quand les deux parents sont bascophones, mais chute fortement lorsqu'uniquement l'un des géniteurs maîtrise cette langue. De même, si la transmission habituelle se situe légèrement au dessus des $20 \%$, la transmission occassionnelle dépasse les $10 \%$ auprès des jeunes générations.

2) Dès lors, il apparaît que l'érosion de la transmission de la langue basque s'est arrêtée. Ainsi, la transmission habituelle s'est stabilisée, surtout en ce qui concerne les personnes nées entre 1965 et 1975 qui se situe à $21 \%$. Alors que cette transmission a connu une forte chute passant de plus de $40 \%$ pour les personnes nées dans les années 1920 à $20 \%$ pour celles qui ont vu le jour en 1955. Par contre, la transmission occasionnelle croît sensiblement puisqu'elle double en cinq ans passant de $6 \%$ pour les personnes nées en 1970 à $11 \%$ pour celles qui le sont en 1975. Au total, la transmission s'améliore auprès des nouvelles générations, bien que ce soit avant tout au profit de la transmission occassionnelle. 
Cette meilleure transmission résulte d'un début de prise de conscience, suite à la création puis à l'organisation d'un mouvement culturel en faveur du soutien et de la promotion de la langue basque. Ainsi, diverses associations oeuvrant pour le basque ont vu le jour puis se sont regroupés au sein de fédérations, ayant pour nom Pizkundea puis Euskal Konfederazioa. Elles soulignent la nécessité de sauvegarder le basque en tant que langue en difficulté.

\section{Retransmission de la langue basque par génération}

\begin{tabular}{|c|c|c|c|c|c|c|c|}
\hline \multirow[b]{3}{*}{ Année } & \multicolumn{7}{|c|}{$\begin{array}{l}\text { Nombre de locuteurs adultes parlant basque à leurs enfants alors que leurs parents parłaient } \\
\text { habituellement la langue basque }\end{array}$} \\
\hline & \multicolumn{2}{|c|}{ Habituellement } & \multicolumn{2}{|c|}{ Occasionnellement } & \multicolumn{2}{|c|}{ Jamais } & \multirow[b]{2}{*}{ Population } \\
\hline & Effectifs & $\%$ & Effectifs & $\%$ & Effectifs & $\%$ & \\
\hline$1 9 \longdiv { 1 5 }$ & 2046 & 20,9 & 590 & 6,0 & 7173 & 73,1 & 9809 \\
\hline 1920 & 3061 & 26,7 & 476 & 5,2 & 7929 & 69,1 & 11466 \\
\hline 1925 & 2654 & 20,2 & 586 & 4,5 & 9896 & 75,3 & 13136 \\
\hline 1930 & 3495 & 22,9 & 733 & $\overline{4,8}$ & II 019 & 72,3 & 15247 \\
\hline 1935 & 2589 & 18,4 & 846 & 6,0 & 10652 & 75,6 & 14087 \\
\hline 1940 & 1598 & 14,5 & 685 & 6,2 & $\overline{8725}$ & 79,3 & 11008 \\
\hline 1945 & 2298 & 13,0 & 749 & 4,2 & 14638 & 82,8 & 17685 \\
\hline 1950 & 2695 & 15,4 & 678 & 3,9 & 14180 & 80,8 & 17553 \\
\hline 1955 & 1582 & 10,1 & 805 & 5,1 & $1 \overline{3} 313$ & 84,8 & 15700 \\
\hline 1960 & 1990 & 12,2 & 1360 & 8,3 & 13017 & 79,5 & 16367 \\
\hline 1965 & 1049 & 8,6 & 1246 & 10,2 & $\overline{9927}$ & 81,2 & 12222 \\
\hline 1970 & 446 & 5,9 & 248 & 3,3 & $6 \overline{815}$ & 90,8 & 7509 \\
\hline $1 \overline{975}$ & - & - & 93 & $2, \overline{9}$ & 3116 & 97,1 & 3209 \\
\hline Total & 25503 & 15,5 & 9095 & 5,5 & 130390 & 79,0 & 164998 \\
\hline
\end{tabular}

Source : Insee - Enquête 1999 Etude de l'histoire familiale

Or, pour que cette volonté puisse se traduire en acte, il est nécessaire que les bascophones jouissent d'un sentiment de compétence linguistique suffisant (car celui-ci conditionne amplement la propension à parler en basque), ce qui est de moins en moins le cas. Ainsi, la proportion de locuteurs bascophones qui déclarent mieux maîtriser le français que le basque est en augmentation ces cinq dernières années alors que ceux qui affirment mieux parler le basque que le français sont moins nombreux.

3) L'on peut raisonnablement penser que la transmission, occasionnelle surtout, se fera de mieux en mieux auprès des nouvelles générations, comme en témoigne l'essor de la demande sociale vis-à-vis de la langue basque. Ainsi, le sondage intitulé "Les parents d'enfants de 0 à 2 ans du BAB et l'apprentissage de la langue basque" montre que $56 \%$ des personnes intérrogées sont disposées à scolariser leurs enfants dans l'enseignement bilingue et que $75 \%$ souhaitent le faire dès la maternelle. Ces chiffres sont d'autant plus significatifs que l'enquête a été réalisée dans l'agglomération bayonnaise qui est la plus urbaine et la moins bascophone. 
Nonobstant, bien souvent, l'offre est inférieure à la demande. L'enseignement bilingue dans le $\mathrm{BAB}$ offre un bon exemple de ce décalage entre une demande sans cesse croissante et une offre insuffisante en établissements, en enseignants, en aides-maternelles et en matériel pédagogique.

\section{Retransmission de la langue basque}

1) La retransmission du basque, c'est-à-dire la transmission de cette langue des parents à leurs enfants après l'avoir reçue de leurs propres pères et mères, est étudiée selon la génération. Elle se situe à un niveau relativement élevée auprès des jeunes personnes et résiste nettement mieux que les autres langues dites régionales.

2) Or, les chiffres révèlent un déclin lent mais régulier de cette retransmission puisque si, pour les personnes nées en $1915,73,1 \%$ des parents connaissant la langue basque ne la transmettaient pas à leurs enfants, $81,2 \%$ en faisaient de même en ce qui concerne celles ayant vu le jour en 1965. Toutefois, une analyse plus précise permet de distinguer deux périodes. La première période regroupe les personnes nées entre 1915 et 1935 puisque l'absence de retransmission est relativement stable oscillant entre $73,1 \%$ et $75,6 \%$. La seconde englobe les personnes ayant vu le jour entre 1940 et 1965 dans la mesure où ce taux reste assez constant autour de $82 \%$ de non retransmission.

De même, il convient de distinguer la retransmission habituelle et occasionnelle, car si la première décline assez régulièrement entre les générations nées en 1915 et en 1970 (à savoir les personnes ayant en 1999 entre 84 et 29 ans), à l'exception des générations de 1960 et de 1950 , il en est tout autrement de la retransmission occasionnelle. Effectivement, cette retransmission a plus que doublé entre les générations de 1920 et de 1960. En réalité, l'on observe un glissement de la retransmission habituelle vers la retransmission occasionnelle, ce qui permet de relativiser l'ampleur de la chute de la retransmission.

3) $\mathrm{Au}$ cours des prochaines années, la retransmission, occasionnelle surtout, devrait continuer à croître avec la présence supérieure du basque dans la vie publique en général et dans les services publics, la signalétique et les médias en particulier. Ainsi, outre la création puis le développement du groupe de communication EITB et des radios d'expression basque, la presse écrite offre l'opportunité de lire en langue basque avec l'avènement d'Euskaldunon Egunkaria et, dans une moindre mesure, du Journal du Pays Basque. De même, la langue basque est de plus en plus visible dans la signalétique interne et externe des communes.

\section{Retransmission de la langue basque suivant la pratique linguistique des parents}

La retransmission du basque diffère selon la pratique linguistique des géniteurs.

\section{Lorsqu'aucun des deux parents ne parlait le basque}


1) Ainsi, quand aucun des pères et mères des parents ne parlait habituellement la langue basque, la transmission à leurs propres enfants a été très faible, généralement inférieure à $5 \%$. Effectivement, les deux dernières générations recensées (les personnes nées en 1970 et en 1965 qui ont en 199929 et 34 ans) ne retransmettent la langue basque qu'à un peu plus de $2 \%$.

\begin{tabular}{|c|c|c|c|c|c|c|}
\hline \multirow[b]{3}{*}{ Année } & \multicolumn{6}{|c|}{$\begin{array}{l}\text { Nombre de locuteurs ayant retransmis la langue basque } \\
\text { à leurs enfants alors qu'aucun des parents ne parlait babituellement } \\
\text { la langue basque }\end{array}$} \\
\hline & \multicolumn{2}{|c|}{ Habituellement } & \multicolumn{2}{|c|}{ Jamais } & \multicolumn{2}{|c|}{ Occasionnellement } \\
\hline & Effectif & $\%$ & Effectif & $\%$ & Effectif & $\%$ \\
\hline 1915 & 33 & 0,5 & 5905 & 97,1 & 147 & 2,4 \\
\hline 1920 & 116 & 1,6 & 7074 & 95,8 & 191 & 2,6 \\
\hline 1925 & 92 & 1,1 & 8493 & 97,4 & 133 & 1,5 \\
\hline 1930 & & & 991 & 97,9 & 211 & 2,1 \\
\hline 1935 & 167 & 1,7 & 9424 & 97,4 & 85 & 0,9 \\
\hline 1940 & & & 7728 & 95,9 & 327 & 4,1 \\
\hline 1945 & 174 & 1,3 & 13034 & 97,3 & 187 & 1,4 \\
\hline 1950 & & & $12\lceil 42$ & 97,8 & 276 & 2,2 \\
\hline 1955 & 125 & 1,0 & 12552 & 98,2 & 104 & 0,8 \\
\hline 1960 & 290 & 2,3 & 12095 & 93,9 & 489 & 3,8 \\
\hline 1965 & 242 & 2,3 & 946 & 89,2 & 901 & 8,5 \\
\hline 1970 & & & 6117 & 97,9 & 132 & 2,1 \\
\hline 1975 & & & 2626 & 96,6 & 93 & 3,4 \\
\hline Total & 1238 & 1,0 & 116559 & 96,3 & 3275 & 2,7 \\
\hline
\end{tabular}

Source : Insee - Enquête 1999 Etude de l'histoire familiale

2) Il est à noter que ce chiffre évolue peu suivant les générations puisque le taux de retransmission reste quasiment inchangé en soixante ans. Nonobstant, une progression lente mais régulière de la retransmission habituelle apparaît puisqu'elle passe de $0,5 \%$ à $2,3 \%$ au cours de la même période. De façon analogue, la retransmission occasionnelle croît pour se situer à $3,4 \%$ pour les personnes nées en 1975.

3) Au cours des prochaines années cette retransmission devrait se développer avec l'essor de la formation du basque pour adultes. Ainsi, les effectifs du principal organisme de formation, AEK en l'occurrence, ont plus que doublé entre 1991 et 1997 en passant de 744 à 1454 élèves. Cette progression est d'autant plus significative que l'enquête sociolinguistique réalisée en 1996 a démontré que les nouveaux bascophones, c'est-à-dire les parents ayant appris cette langue à l'âge adulte, la transmettent mieux à leurs enfants que les vieux bascophones, à savoir, les locuteurs dont le basque est la langue maternelle.

\section{Lorsqu'un des deux parents parlait le basque}

1) Fort logiquement, la retransmission de la langue basque à leurs enfants est supérieure lorsqu'un seul des deux parents parlait habituellement le basque. $\mathrm{La}$ retransmission habituelle est, toute génération confondue, de $17,2 \%$ alors que la 
retransmission occasionnelle est, en moyenne, de $9,7 \%$. Enfin, plus de sept personnes sur dix déclarent ne pas avoir retransmis la langue basque à leurs enfants.

2) L'évolution de la retransmission dans cette configuration est en dents de scie puisque, si la retransmission habituelle est semblable entre les générations nées en 1915 et en 1975, de fortes variations apparaissent entre les personnes ayant vu le jour en $1915(12 \%)$ et en $1920(53,9 \%)$ mais aussi entre les générations de 1960 $(21,5 \%)$ et de $1965(12,3 \%)$. Ces changements ne sont que partiellement imputables aux deux conflits mondiaux.

3) À l'avenir, cette forme de retransmission devrait rester stable, voire croître au fil du temps comme en témoigne le sondage réalisé par l'Institut CSA auprès des parents du $\mathrm{BAB}$ ayant des enfants âgés de 0 à 2 ans. En effet, cette étude révèle que $24 \%$ des parents intérrogés jugent que l'apprentissage du basque est synonyme de culture, d'ouverture d'esprit et d'enrichissement pour l'enfant. De même, $8 \%$ des parents questionnés considèrent que l'acquisition du basque est un atout, un plus.

\section{Lorsque les deux parents parlaient le basque}

1) La retransmission est majeure s'il en est lorsque les deux parents pratiquaient régulièrement cette langue. Dans ce dernier cas, la retransmission habituelle se situe en moyenne autour de 58,2\% et l'occasionnelle se place à 13,5 $\%$. Uniquement dans un peu moins de trois cas sur dix, le basque n'est jamais retransmis. Notons à cet égard, que ce sont les générations nées après et surtout pendant la Première puis la Seconde Guerre Mondiale qui ont le moins retransmis la langue basque à leurs enfants.

\begin{tabular}{|c|c|c|c|c|c|c|}
\hline \multirow[b]{3}{*}{ Année } & \multicolumn{6}{|c|}{$\begin{array}{c}\text { Nombre de locuteurs ayant retransmis la langue basque } \\
\text { à leurs enfants a lors que les deux parents parlaient } \\
\text { habituellement la langue basque }\end{array}$} \\
\hline & \multicolumn{2}{|c|}{ Habituellement } & \multicolumn{2}{|c|}{ Jamais } & \multicolumn{2}{|c|}{ Occasionnellement } \\
\hline & Effectif & $\%$ & Effectif & $\%$ & Effectif & $\%$ \\
\hline 1915 & 1977 & 57,9 & 995 & 29,1 & 443 & 13 \\
\hline 1920 & 2753 & 73,9 & 686 & 18,4 & 285 & 7,6 \\
\hline 1925 & $254 !$ & 61,8 & 112 & 27,2 & 453 & 11 \\
\hline 1930 & 3373 & 69,9 & 930 & 19,3 & 522 & 10,8 \\
\hline 1935 & 2422 & 58,7 & 1007 & 24,4 & 697 & 16,9 \\
\hline 1940 & 1559 & 56,6 & 835 & 30,3 & 358 & 13 \\
\hline 1945 & 2084 & 53,8 & 1225 & 31,7 & 562 & 14,5 \\
\hline 1950 & 2695 & 54,8 & 2039 & 41,4 & 187 & 3,8 \\
\hline 1955 & 1424 & 53,5 & 536 & 20,1 & 701 & 26,3 \\
\hline 1960 & 665 & 50 & 820 & 24,6 & 847 & 25,4 \\
\hline 1965 & 785 & 54,5 & 311 & 21,6 & 346 & 24 \\
\hline 1970 & 446 & 38 & 614 & 52,2 & 116 & 9,8 \\
\hline 1975 & & & 419 & 100 & & \\
\hline Total & 23725 & 58,2 & 11537 & 28,3 & 5517 & 13,5 \\
\hline
\end{tabular}


Source : Insee - Enquête 1999 Etude de l'histoire familiale

2) L'évolution de cette transmission révèle qu'après une baisse relativement regulière, malgré quelques fluctuations, de la retransmission habituelle entre les générations de 1920 et 1945, celle-ci reste stable depuis. En effet, les fluctuations sont inférieures à $1 \%$. En revanche, la transmission occassionnelle, qui connait de fortes variations, progresse globalement pour dépasser les $25 \%$ pour les personnes ayant vu le jour en 1955 et 1960 . Autrement dit, un adulte sur quatre dont les deux parents parlaient habituellement la langue basque la retransmet à son tour à ses enfants, mais de façon occassionnelle.

3) L'on peut raisonnablement penser que la retransmission habituelle se maintiendra, voire croîtra et que la retransmission occasionnelle poursuivra sa progression. D'autant que, l'enquête sociolinguistique de 1996 montre qu'une majorité de parents d'élèves sont favorables au développement de l'enseignement bilingue et que $41 \%$ des personnes intérrogées enverraient leurs enfants dans l'enseignement bilingue s'ils avaient des enfants en âge d'aller à l'école. De même, $63 \%$ estiment que pour intégrer la fonction publique il faudrait savoir parler de basque et $53 \%$ considèrent que toutes les radios et télévisions devraient offrir davantage d'émissions en langue basque.

\section{Conclusion}

Rappelons que cet article se propose d'effectuer un commentaire (diagnostic, évolution, prospective) à partir des données recueillies lors de l'enquête Etude de l'histoire familiale de 1999 réalisée par l'INSEE. Nous soutenons l'idée selon laquelle, après une période de déclin plus ou moins continu, la langue basque semble en voie de stabilisation, voire de réappropriation dans certains domaines tels que la transmission familiale occasionnelle. Or, pour qu'il en soit ainsi dans un avenir plus ou moins proche des mesures ambitieuses de soutien et de promotion de la langue basque devront être adoptées.

\section{Définitions}

Langues régionales : dans cette étude sont considérées comme langues régionales, toutes les langues historiquement parlées sur une partie du territoire métropolitain. $\mathrm{Au}$ Pays Basque, la langue la plus représentative est le basque, bien que cette dernière ait un statut de langue nationale de l'autre côté de la frontière.

Langues étrangères : dans cette catégorie, sont classées toutes les autres langues, même si ce sont des langues locales ou minoritaires et à cheval sur plusieurs pays.

Taux de transmission : proportion de personnes qui ont déclaré habituellement parler à leurs enfants la langue que l'un au moins de leurs parents utilisait habituellement avec eux.

Transmission: les langues parlées par les parents à leurs enfants.

Retransmission : les langues parlées par les parents qui les ont reçues d'au moins l'un des deux parents. 
Réception: les langues que les enfants reçoivent de leurs parents.

\section{Bibliographie}

- Les enquêtes sociolinguistiques du Pays Basque de 1991, 1996 et 2001.

- Atlas des langues en péril dans le monde, UNESCO 2002.

- Le sondage CSA : Les parents d'enfants de 0 à 2 ans du BAB et l'apprentissage de la langue basque, octobre 2001.

- Max Brisson: Analyse du sondage CSA, Commission aménagement linguistique, Conseil des Elus du Pays Basque, 2002.

- Martinez de Luna, Iñaki eta beste : Etorkizuna aurreikusten 99, Euskal Herriko gaztetxoak eta euskara, EHU-UPV, 2000.

- Institut Culturel Basque : Regard sur l'avenir, les adolescents et la langue basque, situation au Pays Basque nord, 2001.

- SEI : Erabileraren IV Kale Neurketa, 2002.

- Statistiques scolaires du primaire, du secondaire et du supérieur entre 1996-2002.

- INSEE : Insee Première, n830, février 2002

- INSEE : "Etude de l'histoire familiale", Courrier des statistiques, n93, 2000.

- INED : "L'unification linguistique de la France". Population et Sociétés, n²85, 1993.

- INED : "La dynamique des langues en France", Population et Sociétés, n³76, 2002. 